\title{
A search for CO in the Local Group dwarf irregular galaxy WLM
}

\author{
C. L. Taylor ${ }^{1,2}$ and U. Klein ${ }^{3}$
}

1 Ruhr-Universität Bochum, Astronomisches Institut, Universitätsstraße 150, 44780 Bochum, Germany

2 Five College Radio Astronomy Observatory, University of Massachusetts, 619B Lederle Research Tower, Amherst, MA 01003, USA

3 Universität Bonn, Radioastronisches Institut, Auf dem Hügel 71, 53121 Bonn, Germany

Received 17 August 2000 / Accepted 28 November 2000

\begin{abstract}
We present ${ }^{12} \mathrm{CO} J=1 \rightarrow 0$ and $J=2 \rightarrow 1$ observations of the low metallicity $(12+\log (\mathrm{O} / \mathrm{H})=7.74)$ Local Group dwarf irregular galaxy WLM made with the $15 \mathrm{~m}$ SEST and $14 \mathrm{~m} \mathrm{FCRAO} \mathrm{telescopes.} \mathrm{Despite} \mathrm{the}$ presence a number of HII regions, we find no $\mathrm{CO}$ emission. We obtain low upper limits on the integrated intensity $\left(I_{\mathrm{CO}} \leq 0.18 \mathrm{~K} \mathrm{~km} \mathrm{~s}^{-1}\right.$ for $\left.\mathrm{CO}(1 \rightarrow 0)\right)$. The non-detection is consistent with the result of Taylor, Kobulnicky \& Skillman (1998), that dwarf galaxies below a metallicity of $\sim 7.9$ are not detected in CO emission. WLM shows that this trend continues for low metallicity galaxies even as their metallicities approach 7.9. These results are consistent with the models of the metal poor ISM by Norman \& Spaans (1997). By comparing our CO data with observations of star formation in WLM, we find evidence for a high $\mathrm{CO}$ to $\mathrm{H}_{2}$ conversion factor.
\end{abstract}

Key words. ISM: molecules - galaxies: individual: WLM - galaxies: ISM - radio lines: galaxies

\section{Introduction}

The WLM galaxy is a dwarf irregular that sits on the edge of the Local Group. Its distance is $0.9 \mathrm{Mpc}$ (Minniti \& Zulstra 1996), close enough for detailed studies of the stellar populations both from the ground (Minniti \& Zulstra 1996) and from HST (Dolphin 2000). As is typical for dwarf galaxies, the metallicity of WLM is low, $12+$ $\log (\mathrm{O} / \mathrm{H})=7.74$ (Skillman et al. 1989), while there is a comparatively large amount of atomic hydrogen $\left(M_{\mathrm{HI}}=\right.$ $4.710^{7} M_{\odot}$; Huchtmeier et al. 1981). Morris \& Lo (1978) observed a single position at the center of the galaxy in the $\mathrm{CO} J=1 \rightarrow 0$ line, obtaining a $3 \sigma$ upper limit of $0.25 \mathrm{~K}$.

$\mathrm{CO}$ has been difficult to detect in dwarf galaxies, a fact which is now most often attributed to their low metal content (e.g. Taylor et al. 1998). Taylor et al. (1998) found evidence for a dependency of the integrated CO $J=1 \rightarrow 0$ line intensity, $I_{\mathrm{CO}}$, upon metallicity, such that only dwarfs with metallicities greater than $\sim 8$ on the $12+\log (\mathrm{O} / \mathrm{H})$ scale were detected, even with extremely low upper limits obtained on the more metal poor galaxies. The metallicity of WLM places it between the detected galaxies and the more metal poor ones, making it a logical candidate for a new search for $\mathrm{CO}$ emission. The tremendous improvements in receiver technology since the 1970's allow for much lower upper limits than could be obtained by Morris \& Lo (1978) and for observations of multiple

Send offprint requests to: C. L. Taylor positions within the galaxy. Here we report on our search for CO emission in the WLM dwarf irregular galaxy.

\section{Observations and data reduction}

\subsection{SEST}

WLM was observed using the $15 \mathrm{~m}$ Swedish ESO Submillimeter Telescope (SEST) at La Silla, Chile, between 1999 January 10-16. Observations were carried out simultaneously in the $\mathrm{CO} J=1 \rightarrow 0$ line at $115 \mathrm{GHz}$ and the $J=2 \rightarrow 1$ line at $230 \mathrm{GHz}$. The beamsize at $115 \mathrm{GHz}$ is $45^{\prime \prime}$, and at $230 \mathrm{GHz}$ is $23^{\prime \prime}$. Using the Low Resolution Spectrometers, velocity resolutions of $1.8 \mathrm{~km} \mathrm{~s}^{-1}$ and $0.9 \mathrm{~km} \mathrm{~s}^{-1}$ were obtained at $115 \mathrm{GHz}$ and $230 \mathrm{GHz}$, respectively. The receivers were tuned to the systemic velocity of the galaxy, $-116 \mathrm{~km} \mathrm{~s}^{-1}$. The system temperatures varied from 330 to $470 \mathrm{~K}$ at $115 \mathrm{GHz}$ and from 250 to $420 \mathrm{~K}$ at $230 \mathrm{GHz}$. The pointing was checked at the beginning of each observing session by observing the nearby $\mathrm{SiO}$ maser source $\mathrm{R}$ Aqr.

Observations were made of seven positions within the galaxy, each in proximity to the groups of HII regions observed by Hodge \& Miller (1995). In other Local Group dwarf irregulars, such as IC 10 and NGC 6822, CO emission has been found in the vicinity of HII regions (e.g. Wilson 1992; Wilson 1995). 


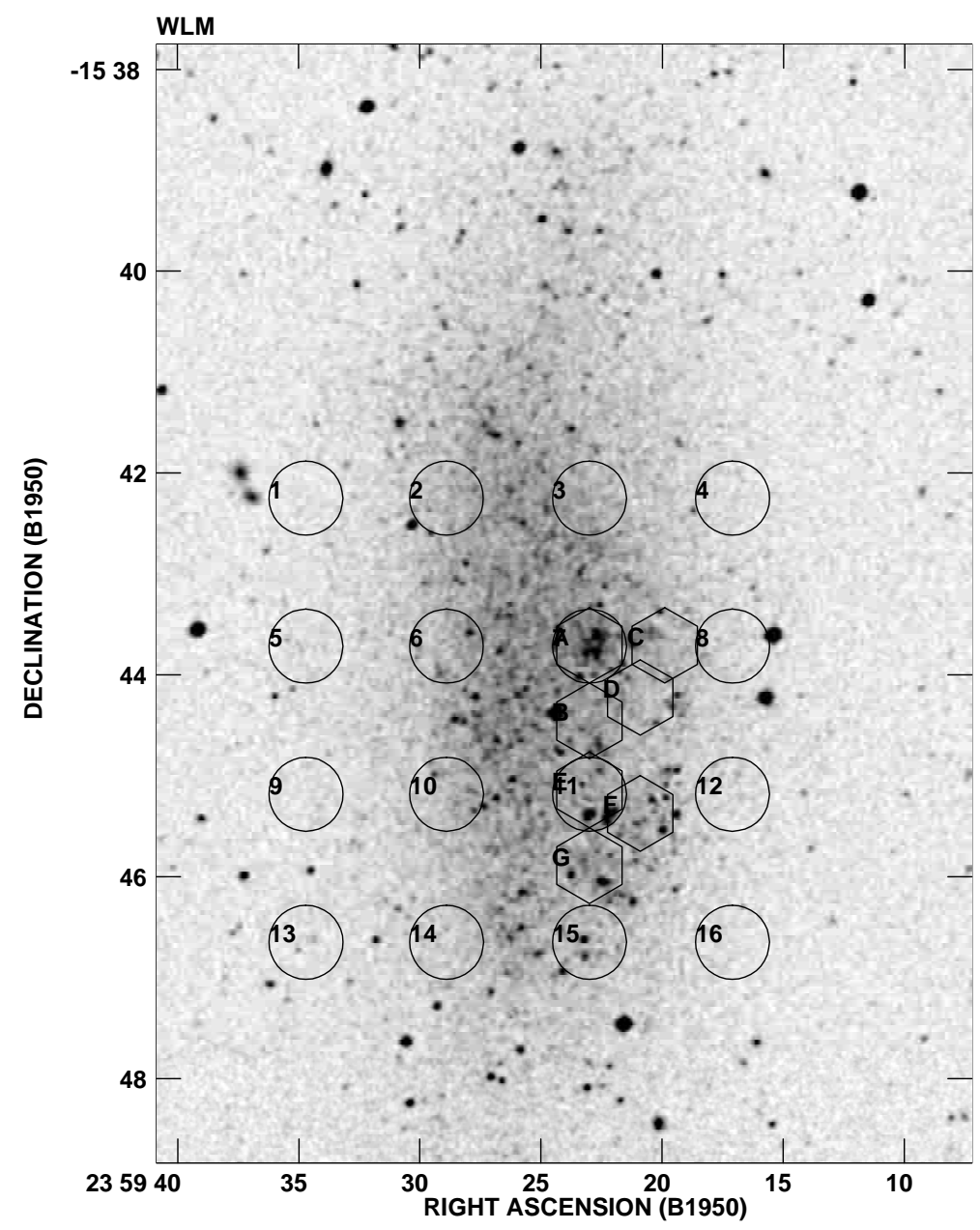

Fig. 1. Location of the SEST (hexagons) and FCRAO (circles) superposed over a digitized POSS image of WLM

\subsection{FCRAO}

Observations were also obtained with the Five College Radio Astronomy Observatory $14 \mathrm{~m}$ telescope in New Salem, Massachusetts from 1-3 December 1999, 30 December 1999-2 January 2000 and 10-14 February 2000. The SEQUOIA focal plane array receiver, consisting of 16 pixels operating in the 85 to $115 \mathrm{GHz}$ band, was used to observe the $\mathrm{CO} J=1 \rightarrow 0$ line. The beamsize of the telescope at $115 \mathrm{GHz}$ is $44^{\prime \prime}$, and the spacing between pixels in the array is $88^{\prime \prime}$. The Focal Plane Array Autocorrelation Spectrometers (FAAS) were used to obtain a bandwidth of $80 \mathrm{MHz}$ with $313 \mathrm{kHz}\left(0.8 \mathrm{~km} \mathrm{~s}^{-1}\right)$ channels. Because of the southern declination of WLM the system temperatures were higher in the FCRAO data than in the SEST data, ranging from $\sim 600$ to $1000 \mathrm{~K}$. The pointing was checked every 2 hours each session by observing the $\mathrm{SiO}$ maser sources $\mathrm{R}$ Cas and $\mathrm{T}$ Ceph.

The telescope was pointed so that the central 2 columns of 4 pixels in SEQUOIA fell within the optical galaxy. The outer two columns, although outside most of the optical light from WLM, were well within the bounds of the HI distribution (Huchtmeier et al. 1981). Pixel 11 in the array was aimed to point at one of the prominent groups of HII regions identified by Hodge \& Miller (1995).
The telescope was operated in a staring mode, where the position was not changed to fill in the gaps between the SEQUOIA pixels.

The SEQUOIA observations are complementary with the SEST data because they are not biased to observing only in the vicinity of HII regions. While HII regions are an indication of recent massive star formation, and hence likely places to find molecular gas, $\mathrm{CO}$ emission can be found in areas with no corresponding $\mathrm{H} \alpha$ emission (e.g. IC 10, Becker 1990; NGC 4214, Walter et al. 2000). Figure 1 shows the location of the SEST and FCRAO observations compared to an optical image of WLM.

\subsection{Data reduction}

The data were reduced using the CLASS package. We subtracted a linear baseline from each individual scan; scans for which a linear baseline was not sufficient were discarded. The final spectra for each position were obtained by averaging all the scans at each position. To decrease the rms noise in the final SEST spectra, we smoothed them to velocity resolutions of 7.2 and $16.2 \mathrm{~km} \mathrm{~s}^{-1}$ for the $1 \rightarrow 0$ data and to 2.7 and $7.3 \mathrm{~km} \mathrm{~s}^{-1}$ for the $2 \rightarrow 1$ data. To convert to the main beam temperature scale, 
Table 1. SEST positions in WLM

\begin{tabular}{|c|c|c|c|c|c|c|}
\hline Position & $\begin{array}{c}\Delta \mathrm{RA} \\
(\operatorname{arcsec})\end{array}$ & $\begin{array}{c}\Delta \mathrm{Dec} \\
(\operatorname{arcsec})\end{array}$ & $\begin{array}{c}\sigma\left(T_{\mathrm{mb}}\right)(1-0) \\
(\mathrm{K})\end{array}$ & $\begin{array}{c}I_{\mathrm{CO}}(1-0) \\
\left(\mathrm{K} \mathrm{km} \mathrm{s}^{-1}\right)\end{array}$ & $\begin{array}{c}\sigma\left(T_{\mathrm{mb}}\right)(2-1) \\
(\mathrm{K})\end{array}$ & $\begin{array}{c}I_{\mathrm{CO}}(2-1) \\
\left(\mathrm{K} \mathrm{km} \mathrm{s}^{-1}\right)\end{array}$ \\
\hline $\mathrm{A}$ & 0 & 0 & 0.005 & $<0.18$ & 0.003 & $<0.11$ \\
\hline B & 0 & -45 & 0.004 & $<0.14$ & 0.003 & $<0.11$ \\
\hline $\mathrm{C}$ & -45 & 0 & 0.004 & $<0.14$ & 0.003 & $<0.11$ \\
\hline $\mathrm{D}$ & -31 & -31 & 0.004 & $<0.14$ & 0.003 & $<0.11$ \\
\hline $\mathrm{E}$ & 0 & -86 & 0.009 & $<0.32$ & 0.005 & $<0.18$ \\
\hline $\mathrm{F}$ & -31 & -100 & 0.004 & $<0.14$ & 0.003 & $<0.11$ \\
\hline $\mathrm{G}$ & 0 & -131 & 0.004 & $<0.14$ & 0.002 & $<0.07$ \\
\hline
\end{tabular}

Note: The $(0,0)$ position is $23^{\mathrm{h}} 59^{\mathrm{m}} 23.0^{\mathrm{s}},-15^{\circ} 43^{\prime} 42.5^{\prime \prime}$.

Table 2. FCRAO positions in WLM

\begin{tabular}{lcclcc}
\hline Position & $\begin{array}{c}\sigma\left(T_{\mathrm{mb}}\right)(1-0) \\
(\mathrm{K})\end{array}$ & \begin{tabular}{c}
$I_{\mathrm{CO}(1-0)}\left(\mathrm{K} \mathrm{km} \mathrm{s}^{-1}\right)$ \\
\hline 1
\end{tabular} & $\begin{array}{c}\text { Position } \\
2\left(T_{\mathrm{mb}}\right)(1-0) \\
(\mathrm{K})\end{array}$ & $\begin{array}{c}I_{\mathrm{CO}}(1-0) \\
\left(\mathrm{K} \mathrm{km} \mathrm{s}^{-1}\right)\end{array}$ \\
\hline 2 & 0.011 & $<0.40$ & 9 & 0.016 & $<0.58$ \\
4 & 0.009 & $<0.32$ & 10 & 0.013 & $<0.47$ \\
5 & 0.011 & $<0.40$ & 11 & 0.009 & $<0.32$ \\
6 & 0.007 & $<0.25$ & 12 & 0.016 & $<0.58$ \\
7 & 0.011 & $<0.40$ & 13 & 0.011 & $<0.40$ \\
8 & 0.013 & $<0.47$ & 14 & 0.013 & $<0.47$ \\
\hline
\end{tabular}

Note: Position 11 corresponds to $23^{\mathrm{h}} 59^{\mathrm{m}} 23.0^{\mathrm{s}},-15^{\circ} 45^{\prime} 10.9^{\prime \prime}$.

main beam efficiencies $\left(\eta_{\mathrm{MB}}\right)$ of 0.70 for $115 \mathrm{GHz}$ and 0.5 for $230 \mathrm{GHz}$ (SEST Handbook) were used. The FCRAO spectra were smoothed to $7.2 \mathrm{~km} \mathrm{~s}^{-1}$, and $\eta_{\mathrm{MB}}=0.45$ was used. All the analysis was done using the spectra at $7.2 \mathrm{~km} \mathrm{~s}^{-1}$ resolution.

\section{Results}

Both the original resolution and the smoothed data were searched for $\mathrm{CO}$ emission. In neither the SEST data at either frequency, nor the FCRAO $115 \mathrm{GHz}$ data was any emission detected. Table 1 gives the properties of the SEST data from the individual positions, including the offset in arcseconds from the center position, the rms noise in the spectra, and the $5 \sigma$ upper limit on integrated intensity $\left(I_{\mathrm{CO}}\right)$, for both the $(1 \rightarrow 0)$ and $(2 \rightarrow 1)$ data. Table 2 gives the rms noise and $\left(I_{\mathrm{CO}}\right) 5 \sigma$ upper limits for the FCRAO data. In all cases the upper limits are calculated assuming undetected emission at the $5 \sigma$ level in one $7.2 \mathrm{~km} \mathrm{~s}^{-1}$ channel.

\section{Discussion}

To compare our WLM data with other gas rich dwarf galaxies, in Fig. 3 we reproduce the plot of $I_{\mathrm{CO}}$ versus galaxy metallicity from Taylor et al. (1998), with a data point for WLM added. The data point we added represents position A from the SEST observations. This was chosen because its spectrum is typical, being neither the lowest nor highest noise, and because this location coincides with large HII regions, and so is a likely place to have expected
$\mathrm{CO}$ emission. Taylor et al. noticed in their data a trend for lower metallicity galaxies to be undetected while those of higher metallicity were detected. This was not a new result, but because they used a relatively large number of galaxies, they found that the division between detected and undetected galaxies occurred at $12+\log (\mathrm{O} / \mathrm{H}) \sim 7.9$ (about 1/10 solar abundance). WLM falls just below this metallicity at 7.74 and in a region in the figure with a dearth of data points. Our non-detection of WLM at the level of $I_{\mathrm{CO}}<0.18 \mathrm{~K} \mathrm{~km} \mathrm{~s}^{-1}$ in $\mathrm{CO}(1 \rightarrow 0)$ reinforces this result. Our new data point shows that the trend for galaxies with metallicities below 7.9 to be undetected in CO emission continues almost right up to a metallicity of 7.9 .

Norman \& Spaans (1997) have studied the formation of molecular gas at high redshifts, where the metallicity is low. They find that the cold, dense phase of the ISM does not form until a metallicity of approximately 0.03 to $0.1 Z_{\odot}$. In their models, before this time star formation occurs at very low levels. At this metallicity C, O, and CO become abundant enough to contribute to cooling the gas, and giant molecular clouds can form. Because dust formation requires metals, it is also in this metallicity range that there is sufficient dust to shield molecular gas. Also, the amount of molecular gas increases because of the formation of $\mathrm{H}_{2}$ on the surface of dust grains. Although they made their models with protogalactic disks (presumably becoming spiral and elliptical galaxies with evolution), the phase evolution of the ISM broadly fits what is known about dwarf galaxies. Dwarf galaxies exhibit the lowest 

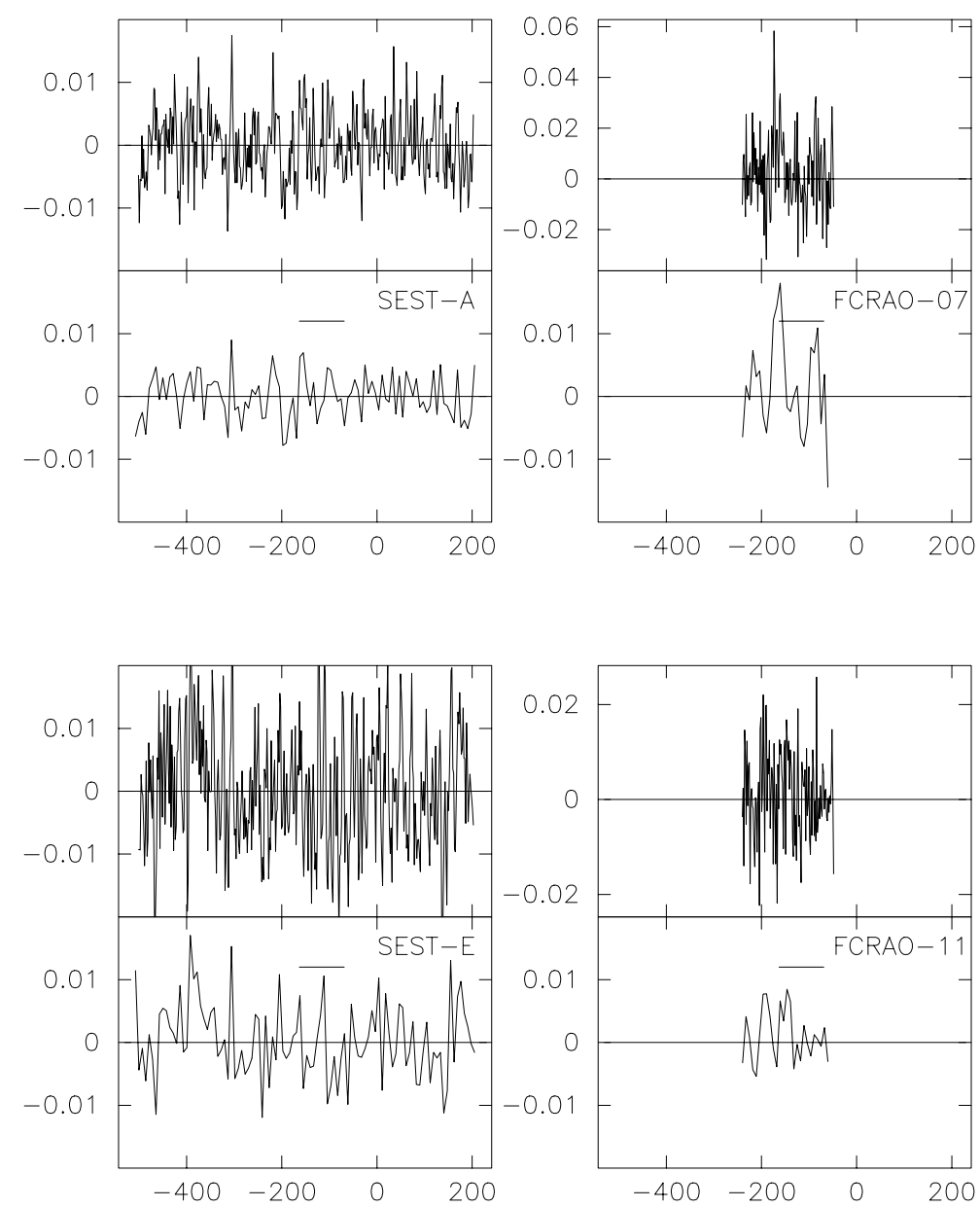

Fig. 2. Example spectra from each telescope. The upper spectra show the original velocity resolution, while the lower are smoothed to $7.2 \mathrm{~km} \mathrm{~s}^{-1}$. The horizontal line shows the full width at $20 \%$ of maximum velocity range of the HI line from Huchtmeier et al. (1981). SEST-A and FCRAO-07 are the same location, as are SEST-E and FCRAO-11

metallicities known from emission line regions, down to $Z \leq 0.03 Z_{\odot}$ (e.g. I Zw 18; Skillman \& Kennicutt 1993). The lowest metallicities seen in these galaxies are close to the lower range of metallicity at which Norman \& Spaans argue that GMCs can form. This would make sense if large star formation episodes can only occur in gas cool enough and dense enough that it is molecular. Our non-detection of WLM agrees with the Norman \& Spaans models as well. The upper portion of the range 0.03 to $0.1 Z_{\odot}$ corresponds to $12+\log (\mathrm{O} / \mathrm{H}) \simeq 8.0$, the metallicity at which dwarf galaxies are no longer detected in $\mathrm{CO}$ in Fig. 3. The metallicity of WLM, 7.74, is below this level.

Taylor et al. (1998) attributed the non-detection of dwarfs at metallicities below $12+\log (\mathrm{O} / \mathrm{H}) \simeq 8.0$ to the $\mathrm{CO}$ to $\mathrm{H}_{2}$ conversion factor $\left(X_{\mathrm{CO}}\right)$ having a dependence on metallicity which becomes sharply non-linear at this metallicity. However, there are not enough data points in Fig. 3 to say if there is truly a sharp cutoff in the detectability, or if the small numbers make it appear so. Clearly more observations, especially of galaxies with metallicities in the range $7.8 \leq 12+\log (\mathrm{O} / \mathrm{H}) \leq 8.0$, are needed before the behavior of $\mathrm{CO}$ emission at low metallicities can be understood.
Verter \& Hodge (1995) obtained very low upper limits on $\mathrm{CO}$ emission in the nearby dwarf galaxy GR 8 , and compared these limits to the $\mathrm{H}_{2}$ column density $\left(N_{\mathrm{H}_{2}}\right)$ inferred from the presence of star formation in the form of HII regions. We will apply their analysis to WLM, using the $\mathrm{H} \alpha$ data of Hodge \& Miller (1995):

$N_{\mathrm{H}_{2}}=9.510^{-23} \frac{L_{\mathrm{H} \alpha}\left(\mathrm{erg} \mathrm{s}^{-1}\right) \tau_{\mathrm{SF}}(\mathrm{yr})}{\epsilon_{\mathrm{SF}} R_{\mathrm{mc}}^{2}(\mathrm{pc})}$

where $L_{\mathrm{H} \alpha}$ is the $\mathrm{H} \alpha$ luminosity, $\tau_{\mathrm{SF}}$ is the timescale of star formation, $\epsilon_{\mathrm{SF}}$ is the star formation efficiency, and $R_{\mathrm{mc}}$ is the radius of a molecular cloud. For $\tau_{\mathrm{SF}}$ we adopt $10^{8} \mathrm{yr}$, as did Verter \& Hodge. This number ultimately derives from Lo et al. (1993), who argue that $\tau_{\mathrm{SF}}$ must be between the cloud-cloud collision time scale (the timescale on which the gas will collapse to form stars) and the timescale for disruption of the ISM by OB stars. The former has an upper limit based upon the crossing time, which for dwarfs is $\sim 210^{8} \mathrm{yr}$, while latter will take place on a time scale of a few $\times 10^{7} \mathrm{yr}$. Verter \& Hodge assumed $\epsilon_{\mathrm{SF}}=1$ to obtain lower limits on $N_{\mathrm{H}_{2}}$, but a more accurate value is 0.02 (e.g. Taylor et al. 1999 for the post-starburst dwarf galaxy NGC 1569, Wilson \& Matthews 1995 for the giant HII regions NGC 604 and NGC 595 in M 33). We will estimate 


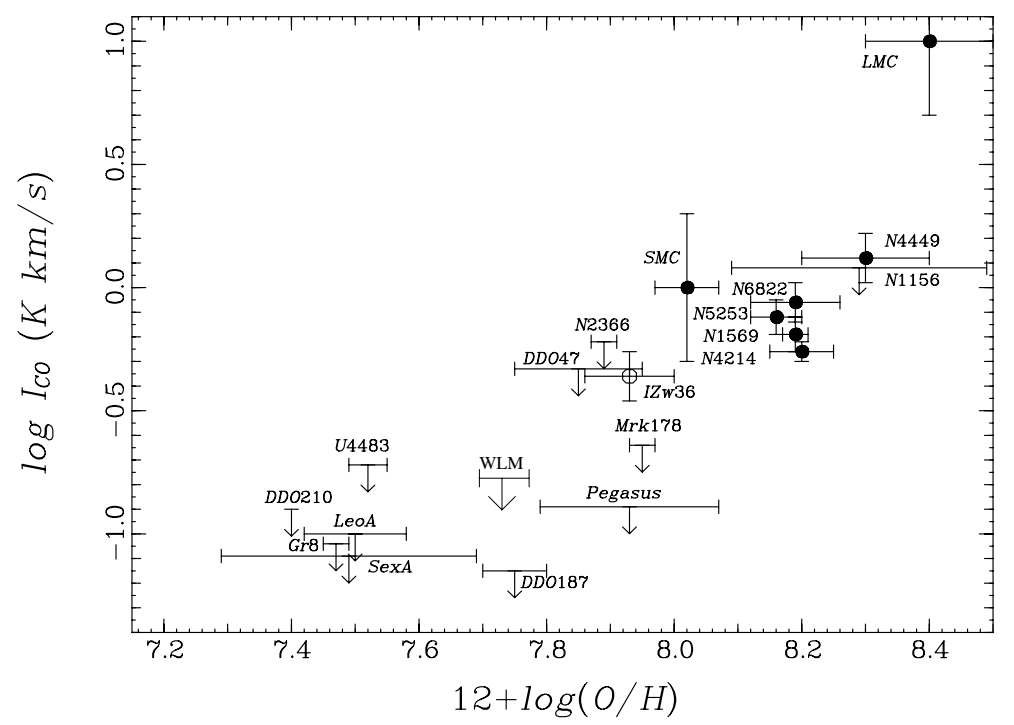

Fig. 3. $\log \left(I_{\mathrm{CO}}\right)$ vs. oxygen abundance, $12+\log (\mathrm{O} / \mathrm{H})$. The plot is reproduced from Taylor et al. (1998), with the data point for WLM from this paper added

the column density with both efficiencies since this is the least secure property used in the calculation. For $R_{\mathrm{mc}}$ we adopt $10 \mathrm{pc}$ based upon spatially resolved observations of giant molecular clouds in nearby dwarf galaxies with interferometers (e.g. IC 10, Wilson \& Reid 1991; Wilson 1995; NGC 6822, Wilson 1994).

We can also estimate $N_{\mathrm{H}_{2}}$ from our CO data, if we assume a $\mathrm{CO}$ to $\mathrm{H}_{2}$ conversion factor. For this we apply the conversion factor - metallicity relation derived by Wilson (1995) from Local Group galaxies, extrapolating down to the metallicity of WLM. This gives a conversion factor of $1.310^{21} \mathrm{~cm}^{-2}\left(\mathrm{~K} \mathrm{~km} \mathrm{~s}^{-1}\right)^{-1}$, or 5.8 times greater than the Galactic value. Note that if the conversion factor behaves as suggested by Taylor et al. (1998), then the true conversion factor could be much higher than this estimate. Therefore, our $N_{\mathrm{H}_{2}}$ estimates should be considered lower limits.

In Table 3 we give the $\mathrm{H} \alpha$ luminosity, the $\mathrm{H}_{2}$ column density estimated from star formation $\left(N_{\mathrm{H}_{2}}(\mathrm{SF})\right)$ for $\epsilon_{\mathrm{SF}}$ $=0.02$ and 1.0, and the $\mathrm{H}_{2}$ column density lower limit from the $\mathrm{CO}$ observations $\left(N_{\mathrm{H}_{2}}(\mathrm{CO})\right)$ for the four SEST positions which overlap with HII regions from Hodge \& Miller (1995). For each position, the limits based on the CO data are already lower than the densities inferred from the star formation. This implies that the conversion factor is even higher than the extrapolation of the Wilson (1995) relation would suggest. In order to get the average $N_{\mathrm{H}_{2}}(\mathrm{CO})$ up to the level of the average $N_{\mathrm{H}_{2}}(\mathrm{SF})$ for the $\epsilon_{\mathrm{SF}}=0.02$ case would require a conversion factor more than 1500 times the Galactic value. Clearly this is extreme, and may result from assuming too low a value for $\epsilon_{\mathrm{SF}}$. In the case of $\epsilon_{\mathrm{SF}}=1$ (which gives the minimum amount of molecular material for the observed star formation rate), a conversion factor of $\sim 30$ times Galactic value would be necessary. A more realistic $\epsilon_{\mathrm{SF}}$ would increase this number. Through the same analysis, Verter \& Hodge arrive at a lower limit for the for the conversion factor in
GR 8 of $\sim 30$ times the Galactic value. GR 8 has a lower metallicity than WLM $(12+\log (\mathrm{O} / \mathrm{H})=7.5$ compared to 7.7 ), so in principle the conversion factor ought to be higher than in WLM, but the fact that we obtain the same upper limit here as Verter \& Hodge merely represents that both galaxies have similar levels of star formation and we have reached similar sensitivities in the observations.

Some dwarf galaxies have regions of $\mathrm{CO}$ emission with no corresponding HII regions, areas in a pre-star formation phase (Becker 1990; Walter et al. 2000). Such regions are not as common, however, as cases where the molecular gas and HII regions are in close association. Clearly in the case of a molecular cloud with no star formation, one could not expect to estimate the molecular gas content associated with newly formed stars. The crude calculation we describe above tells us nothing about such clouds. But HII regions in the Galaxy are often associated with molecular gas on large scales, e.g. the Orion region. This is also true of prominent HII regions in dwarf galaxies like IC 10 (Becker 1990), NGC 1569 (Taylor et al. 1999) and NGC 4214 (Walter et al. 2000). It is unlikely that all of the HII regions in WLM have totally disrupted their natal molecular gas, so it is reasonable to attempt to detect it, and to estimate the emission from these clouds based upon the young stars we currently see.

Of course there are large uncertainties in the above calculation: in addition to the star formation efficiency, one must assume an IMF (Verter \& Hodge use a Salpeter IMF), and neglect dust (observations of IC 10 have shown that dwarf irregulars can host considerable dust - Bolatto et al. 2000). Substantial dust obscuration would mean we have underestimated the molecular gas implied by the observed star formation, which would further increase the conversion factor. Our data certainly do not prove a high $\mathrm{CO}-\mathrm{H}_{2}$ conversion factor, but they are consistent with it.

Klein (2000) has recently argued that the conversion factor, $X_{\mathrm{CO}}$, depends upon the cosmic ray flux. He used 
Table 3. Star formation rates and inferred $\mathrm{H}_{2}$ column densities

\begin{tabular}{lcccc}
\hline Position & $L(\mathrm{H} \alpha)$ & $\begin{array}{c}N_{\mathrm{H}_{2}}(\mathrm{SF}) \\
\mathrm{SF}_{\mathrm{SF}}=0.02 \\
\left(10^{21} \mathrm{~cm}^{-2}\right)\end{array}$ & $\begin{array}{c}N_{\mathrm{H}_{2}}(\mathrm{SF}) \\
\left(10^{21} \mathrm{~cm}^{-2}\right)\end{array}$ & $\begin{array}{l}N_{\mathrm{H}_{2}}(\mathrm{CO}) \\
\left(10^{21} \mathrm{~cm}^{-2}\right)\end{array}$ \\
\hline $\mathrm{A}$ & 2.0 & 95 & 1.9 & $>0.24$ \\
$\mathrm{C}$ & 1.0 & 48 & 0.95 & $>0.18$ \\
$\mathrm{E}$ & 0.8 & 38 & 0.76 & $>0.42$ \\
$\mathrm{G}$ & 2.1 & 100 & 2.0 & $>0.18$ \\
\hline
\end{tabular}

the $6 \mathrm{~cm}$ radio continuum surface brightness as a tracer of cosmic rays and found that as the cosmic ray content increased, $X_{\mathrm{CO}}$ decreased. The implication here is that the heating by cosmic rays provides a significant portion of the energy powering the $\mathrm{CO}$ emission, so that more CO emission per unit $\mathrm{H}_{2}$ mass is observed when the cosmic ray flux is large compared to when it is small. WLM fits into this picture well. It has a $20 \mathrm{~cm}$ continuum flux of only $5 \mathrm{mJy}$ in the NVSS data (Condon et al. 1998), low compared to other dwarf galaxies with measured $20 \mathrm{~cm}$ fluxes (e.g. Höppe et al. 1994; Klein et al. 1991), suggesting a high $X_{\mathrm{CO}}$. The non-detection of $\mathrm{CO}$ is thus not unexpected.

\section{Summary and conclusions}

We have searched for CO emission in the Local Group dwarf irregular galaxy WLM, and found none, obtaining low upper limits on the integrated intensity $\left(I_{\mathrm{CO}} \leq\right.$ $0.18 \mathrm{~K} \mathrm{~km} \mathrm{~s}^{-1}$ for $\left.\mathrm{CO}(1 \rightarrow 0)\right)$. WLM has many HII regions, indicating recent star formation activity, so the lack of $\mathrm{CO}$ emission may indicate a high $\mathrm{CO}$ to $\mathrm{H}_{2}$ conversion factor, rather than a lack of molecular gas. The nondetection is consistent with the result of Taylor et al. (1998) that dwarf galaxies with metallicities less than $12+\log (\mathrm{O} / \mathrm{H}) \simeq 8.0$ are not detected in $\mathrm{CO}$ emission while those with greater metallicities are. This is explained in the models by Norman \& Spaans (1997), who argue that $\mathrm{CO}$ is not abundant in the ISM until metallicities in the range $\sim 0.03$ to $0.1 Z_{\odot}(12+\log (\mathrm{O} / \mathrm{H}) \sim 7.2$ to 7.8$)$. Comparing our upper limits to the molecular gas inferred from the presence of star formation in WLM suggests that the $\mathrm{CO}$ to $\mathrm{H}_{2}$ conversion factor may be as much as 30 times the Galactic value.

Acknowledgements. This work has been supported by the Deutsche Forschungsgemeinschaft under the framework of the Graduiertenkolleg "The Magellanic System, Galaxy
Interaction, and the Evolution of Dwarf Galaxies". The Five College Radio Astronomy Observatory is operated with the permission of the Metropolitan District Commission, Commonwealth of Massachusetts, and with the support of the National Science Foundation under grant AST-9725951.

\section{References}

Becker, R. 1990, Ph.D. Thesis, University of Bonn

Bolatto, A. D., Jackson, J. M., Wilson, C. D., \& MoriartySchieven, G. 2000, ApJ, 532, 909

Condon, J. J., Cotton, W. D., Greisen, E. W., et al. 2000, AJ, 115,1693

Dolphin, A. E. 2000, ApJ, 531, 804

Hodge, P., \& Miller, B. W. 1995, ApJ, 451, 176

Höppe, G., Brinks, E., Klein, U., et al. 1994, AJ, 108, 446

Huchtmeier, W. K., Seiradakis, J. H., \& Materne, J. 1981, A\&A, 102, 134

Klein, U. 2000, in XVIIIth Moriond Astrophysical Meeting, ed. T. X. Thuan, C. Balkowski, V. Cayatte, \& J. Tran Thanh Van (Éditions Frontières: Paris), 107

Klein, U., Weiland, H., \& Brinks, E. 1991, A\&A, 246, 323

Lo, K. Y., Sargent, W. L. W., \& Young, K. 1993, AJ, 106, 507

Minniti, D., \& Zijlstra, A. A. 1996, ApJ, 467, L13

Morris, M., \& Lo, K.-Y. 1978, ApJ, 223, 803

Norman, C. A., \& Spaans, M. 1997, ApJ, 480, 145

Skillman, E. D., \& Kennicutt, R. C. 1993, ApJ, 411, 655

Skillman, E. D., Terlevich, R., \& Melnick, J. 1989, MNRAS, 240,563

Taylor, C. L., Hüttemeister, S., Klein, U., \& Greve, A. 1999, A\&A, 349, 424

Taylor, C. L., Kobulnicky, H. A., \& Skillman, E. D. 1998, AJ, 116,2746

Verter, F., \& Hodge, P. 1995, ApJ, 446, 616

Walter, F., Taylor, C. L., Hüttemeister, S., Scoville, N., \& McIntyre, V. 2001, AJ, in press

Wilson, C. D. 1992, ApJ, 391, L44

Wilson, C. D. 1994, ApJ, 434, L11

Wilson, C. D. 1995, ApJ, 448, L97

Wilson, C. D., \& Matthews, B. C. 1995, ApJ, 455, 125

Wilson, C. D., \& Reid, I. N. 1991, ApJ, 366, L11 